neurosurgeon, and associates at Maudsley Hospital, London, UK, recorded the pathological findings in 100 consecutive surgical patients with temporal lobe epilepsy and found a possible association between mesial temporal sclerosis and febrile convulsions in childhood [2]. Sixty years later we are still uncertain of the exact relationship between temporal lobe epilepsy and febrile seizures. Multiple epileptogenic factors may be involved, including HHV6/HHV7 viremia, developmental delay, and preexisting congenital abnormalities. It is difficult to determine whether HS predates or is the consequence of febrile seizure. As concluded in an invited commentary (Can febrile status cause HS?), the chicken or egg controversy remains unsettled [3]. Of the 9 patients (4\% of 226) who developed changes suggestive of HS, 5 had other MRI abnormalities. Longer follow-up should determine the outcome and occurrence of TLE in this cohort.

\title{
References.
}

1. Jackson GD, et al. Neurology. 1990 Dec;40(12):1869-75.

2. Falconer MA, et al. Arch Neurol. 1964 Mar;10(3):233-48.

3. French JA, Kuzniecky R. Ann Neurol. 2014 Feb;75(2):173-4.

\section{EMERGENCY TREATMENT OF FEBRILE STATUS EPILEPTICUS}

The FEBSTAT study investigators reviewed the charts of 199 patients with FSE, age 1 month to 6 years, recruited from multiple centers from 2002 to 2010 . More than one AED was required to terminate FSE in 140 patients (70\%). Median time from seizure onset to first AED administered by emergency medical services (EMS) or emergency department (ED) was $30 \mathrm{~min}$. Mean seizure duration was $81 \mathrm{~min}$ for subjects who received AED prior to arrival at ED and 95 min for those who did not $(p=0.1)$. Median time from the first dose of AED to the end of seizure was $38 \mathrm{~min}$. Initial dose of lorazepam or diazepam was suboptimal in 32 (19\%) of 166 patients. Respiratory support was required by 95 subjects (48\%). Median seizure duration in the respiratory support group was 83 min whereas for the nonrespiratory support group, seizure duration was 58 $\min (\mathrm{p}<0.001)$. Reducing the time from seizure onset to AED initiation was significantly related to shorter seizure duration. A standard prehospital treatment protocol is recommended with education of EMS responders. (Seinfeld S, Shinnar S, Sun S, et al. Emergency management of febrile status epilepticus: Results of the FEBSTAT study. Epilepsia 2014 Mar;55(3):388-95).

COMMENTARY. EMS care of children participating in FEBSTAT studies varied considerably, some being allowed to administer treatment only when ordered by an ED physician. Delay in AED administration may lead to prolonged FSE and an associated increased risk of respiratory distress. Early treatment of seizures with benzodiazepines does not increase the need for prehospital or ED intubation and respiratory support [1] but rather, results in shorter total seizure duration [2].

\section{References.}

1. Alldredge BK, et al. Pediatr Neurol. 1995 Apr;12(3):213-6.

2. Seinfeld S, et al. Epilepsia. 2014 Mar;55(3):388-95. 